\title{
Endocervical adenocarcinoma: selected diagnostic challenges
}

\author{
Brigitte M Ronnett \\ Departments of Pathology and Gynecology and Obstetrics, The Johns Hopkins Medical Institutions, Baltimore, \\ $M D, U S A$
}

\begin{abstract}
Endocervical adenocarcinomas can be classified into two main types of tumors, namely, those related to highrisk human papillomavirus and those unrelated to high-risk human papillomavirus. The former, representing the vast majority, are referred to as endocervical adenocarcinomas of usual type and the latter are dominated by the gastric-type mucinous adenocarcinomas. Commonly encountered diagnostic problems concerning these endocervical adenocarcinomas include: (1) diagnosing invasion for endocervical adenocarcinomas of usual type, particularly superficial forms which must be distinguished from extensive endocervical adenocarcinoma in situ; (2) distinguishing high-risk human papillomavirus-related endocervical adenocarcinomas from endometrial endometrioid carcinomas; and (3) distinguishing benign/hyperplastic mucinous endocervical glandular proliferations from gastric-type mucinous endocervical adenocarcinomas, particularly minimal deviation adenocarcinoma. The current review provides practical points and numerous illustrative examples to guide pathologists in addressing these diagnostic challenges in routine practice.

Modern Pathology (2016) 29, S12-S28; doi:10.1038/modpathol.2015.131
\end{abstract}

Endocervical adenocarcinomas can be classified into two main types of tumors, namely, those related to high-risk human papillomavirus (HPV) and those unrelated to high-risk HPV. The former, which represent the vast majority, are referred to as endocervical adenocarcinomas of usual type and the latter are dominated by the gastric-type mucinous adenocarcinomas, which include minimal deviation adenocarcinoma. ${ }^{1-8}$ Both types pose a number of diagnostic challenges for practicing pathologists. The most commonly encountered diagnostic problems include the following: (1) diagnosing invasion for high-risk HPV-related (usual type) endocervical adenocarcinomas of usual type, particularly for the common well differentiated and often superficial forms which must be distinguished from extensive endocervical adenocarcinoma in situ (AIS); (2) distinguishing high-risk HPV-related endocervical adenocarcinomas from endometrial endometrioid carcinomas and their related subtypes, which include those with mucinous and metaplastic-type differentiation; and (3) distinguishing benign mucinous endocervical glandular proliferations, including the various forms of endocervical

Correspondence: Dr BM Ronnett, MD, Department of Pathology, The Johns Hopkins Hospital, Weinberg Building, Room 2242, 401 N. Broadway, Baltimore, MD 21231, USA.

E-mail: bronnett@jhmi.edu

Received 28 August 2015; revised 21 September 2015; accepted 26 September 2015 glandular hyperplasia, from gastric-type mucinous endocervical adenocarcinomas, particularly the very well differentiated minimal deviation type. There are excellent comprehensive reviews and original studies addressing these particular topics and the reader is referred to a selection of these references for the clinicopathologic details provided by a more traditional approach. The current presentation is designed to address these topics based on experience gained over 20 years in a large gynecologic pathology practice with numerous consultation cases concerning these diagnostic challenges, emphasizing practical points and utilizing numerous illustrative examples.

\section{Diagnosing invasion for high-risk HPV-related (usual type) endocervical adenocarcinomas}

High-risk HPV-related endocervical adenocarcinomas can exhibit a variety of patterns of invasion, ranging from those that are easily to generally recognized as invasive (Figure 1) to those that are challenging or exceedingly difficult to distinguish from extensive AIS (Figure 2). The former include the following:

1. Infiltrative/destructive (Figures 1a-d). This is characterized by irregular, interconnected cytologically malignant glands infiltrating stroma, 
typically with a stromal reaction (desmoplasia, edema, inflammation). The earliest form is manifested as individual cells, cell clusters, buds, glands, or solid nests arising from AIS, often with more eosinophilic cytoplasm and more vesicular nuclei with nucleoli.
2. Confluent glandular/cribriform (Figures 1e-h). This pattern is characterized by complex growth in which glandular elements are fused, interconnected, or anastomosing to create a tightly packed glandular, cribriform, or labyrinthine growth, which is beyond what is compatible with the
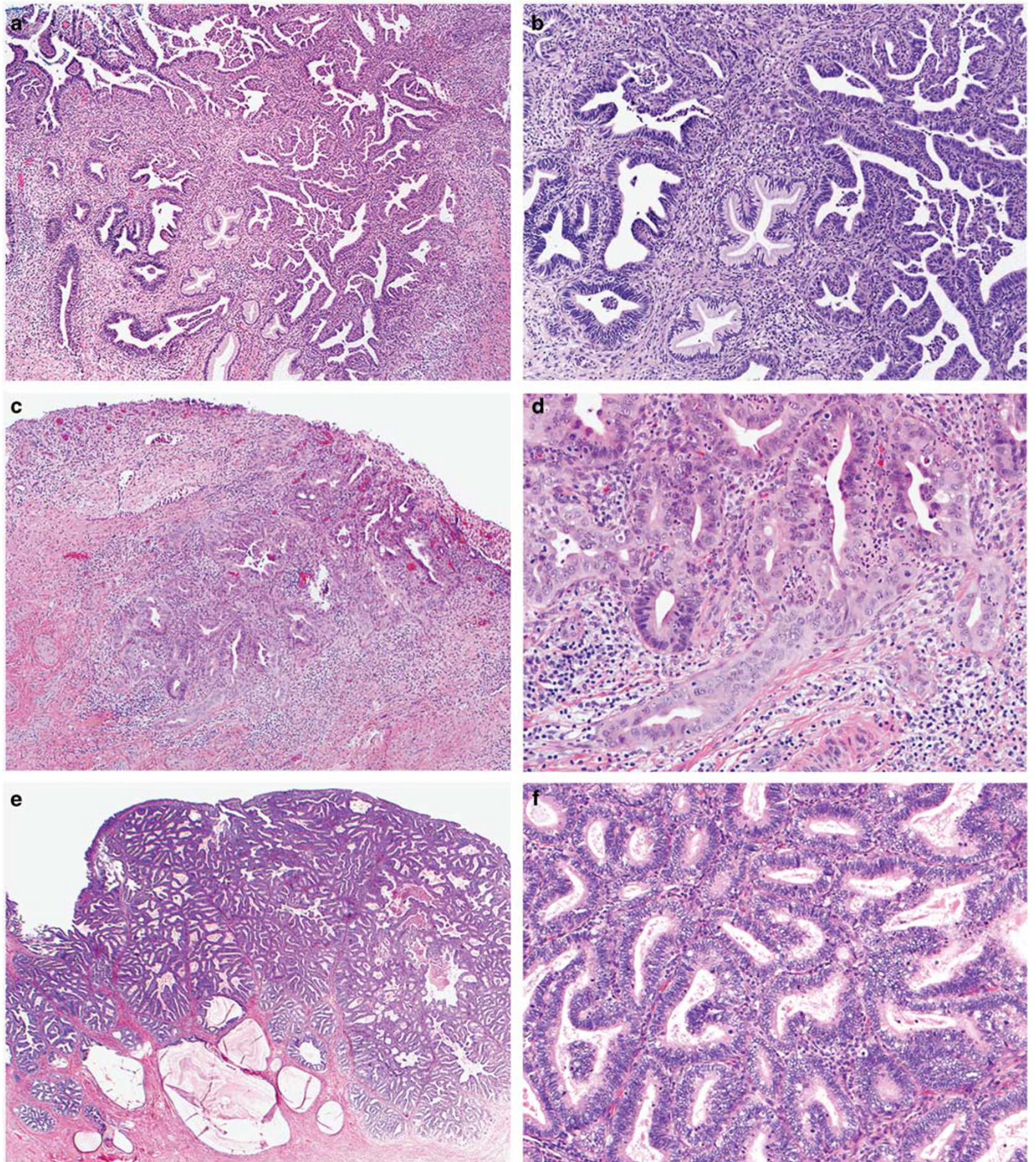

Figure 1 Patterns of invasion in high-risk HPV-related (usual type) endocervical adenocarcinoma: destructive/infiltrative (a-d), confluent glandular (e and f), cribriform ( $\mathbf{g}$ and $\mathbf{h}$ ), and papillary/villoglandular, often exophytic (i and $\mathbf{j}$ ). 

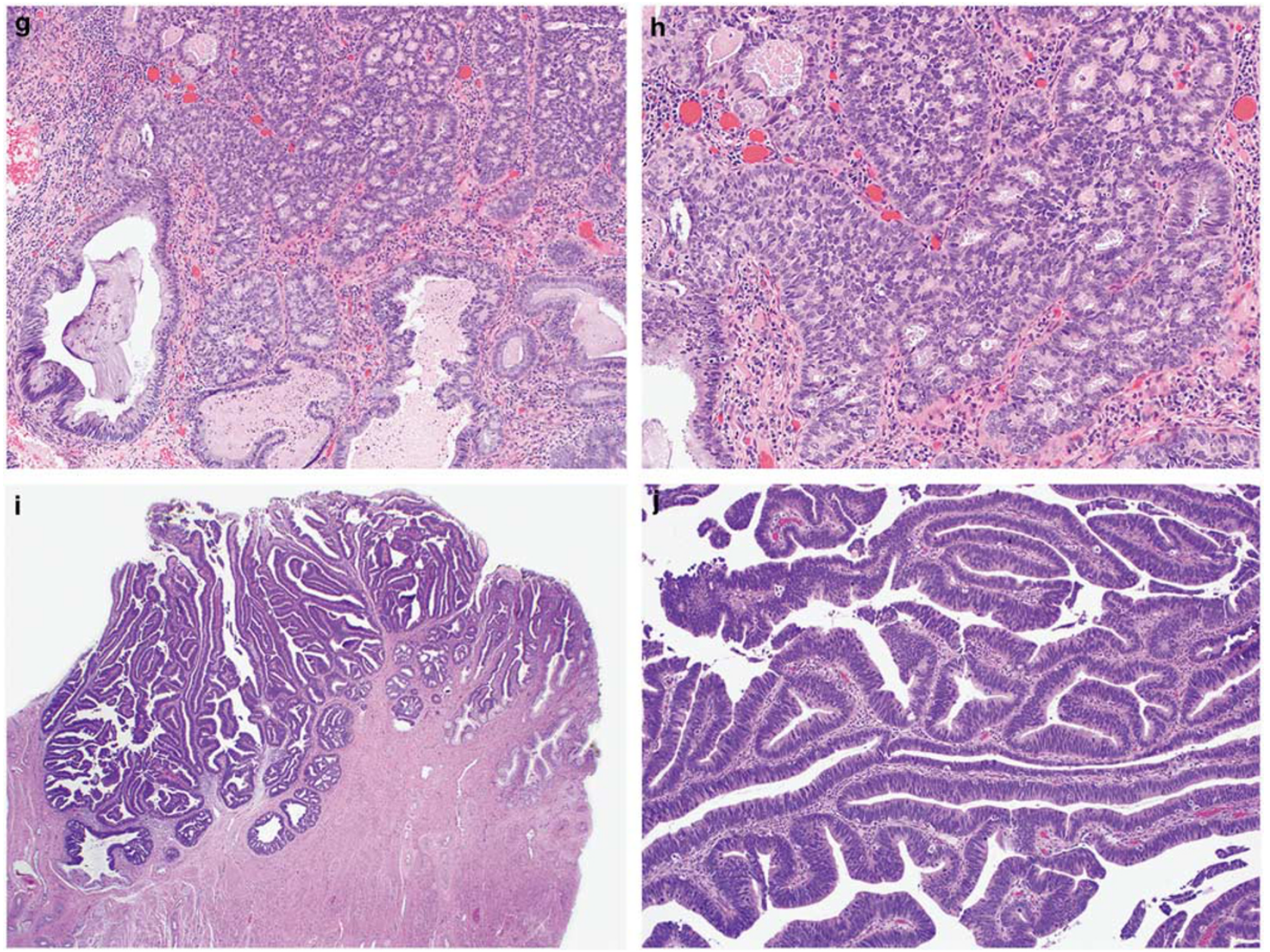

Figure 1 Continued.

normal/background endocervical glandular pattern. Isolated cribriform growth within a single or a few glandular structures is allowed in AIS but interconnection or fusion of such structures indicates an invasive pattern.

3. Papillary/villoglandular (Figures $1 \mathrm{i}$ and j). Tumors with this pattern can be purely exophytic, endophytic, or combination of both, and demonstrate elongated papillary/villous/villoglandular structures, often with some labyrinthine architecture. A purely exophytic form may not invade underlying cervical stroma, but is an invasive adenocarcinoma when its extent is more than just surface AIS with focal papillarity.

Some invasive endocervical adenocarcinomas demonstrate more subtle patterns of invasion, referred to as 'AIS-like' owing to overlap in the growth patterns of these forms of invasion and extensive AIS (Figure 2). ${ }^{9}$ Many are confined to superficial cervical stroma and some are purely surface/exophytic villoglandular proliferations without invasion of underlying cervical stroma, but others can extend deeper into the cervical stroma, with the frequent lack of a stromal reaction making them difficult to distinguish from extensive AIS. These cases suffer from notable interobserver diagnostic variability. Features that are concerning for endocervical adenocarcinoma with early and deceptive patterns of stromal invasion include:

1. A periglandular stromal reaction, which may be rather subtle (lacking typical features of overt desmoplasia; Figures 2a-c).

2. A more extensive, crowded proliferation than the normal/background endocervical glandular pattern, with tight clustering of small glands, early interglandular cribriform growth, and/or early gland fusion (with or without a stromal reaction; Figures 2d-f).

3. Surface exophytic villoglandular growth beyond what is within the spectrum allowed for superficial AIS (only focal/limited papillarity is allowed in the latter; Figures 2g-i).

4. An extensive proliferation that may not be excessively crowded but extends into deeper cervical 
stroma in a haphazard pattern (appearing as if the glands had been strewn across the slide), even if desmoplasia is lacking (Figures $2 \mathrm{j}-\mathrm{l}$ ).
5. Linear tracking of glands into deeper stroma, often in close proximity to thick-walled vessels (Figures 2m-0).
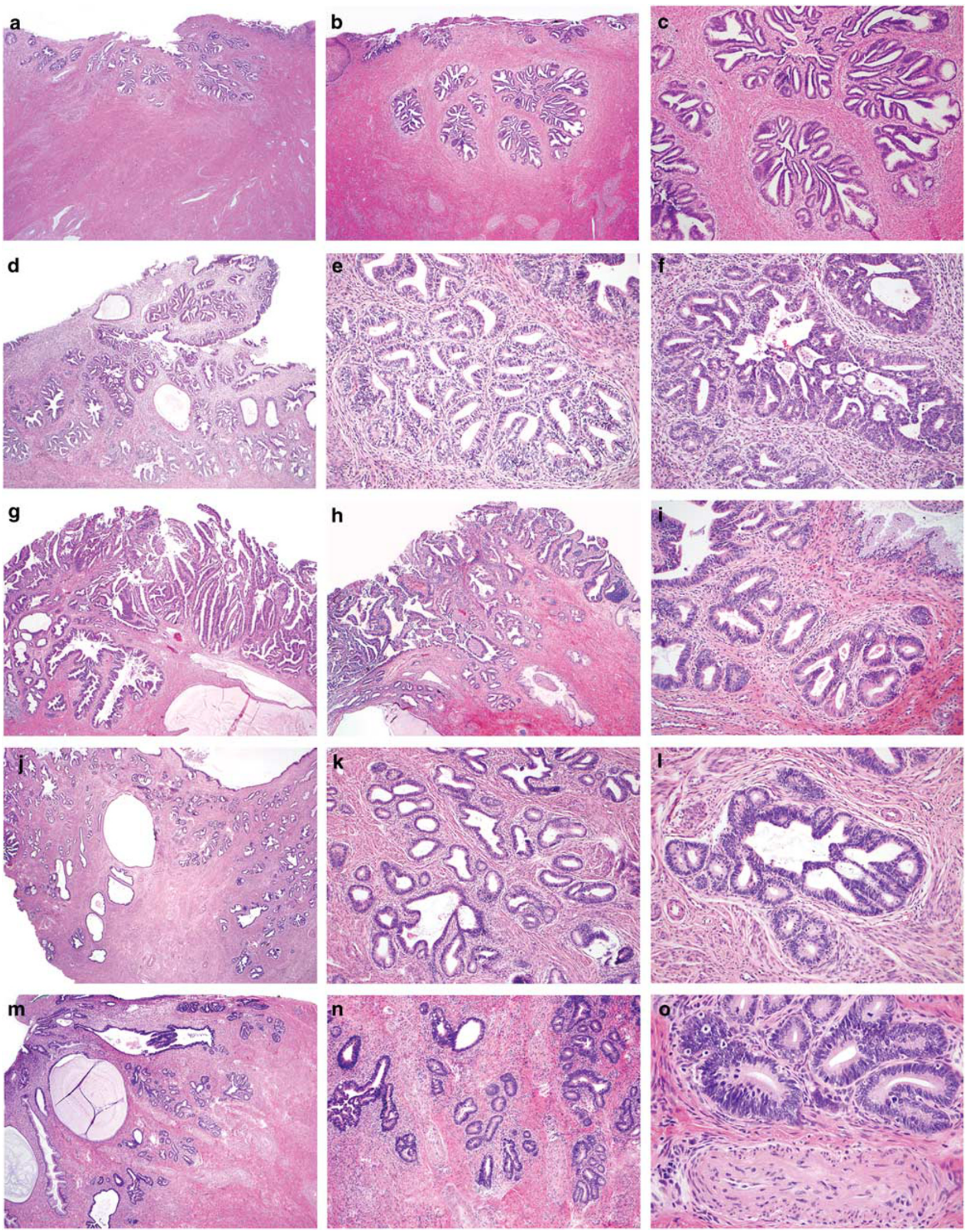

Figure 2 For caption see page S16. 
However, it is often still problematic to unequivocally recognize these features as signs of invasion because the normal glands can be hyperplastic and complex and occasionally can extend deeper into the stroma than usual (so-called deeply penetrating glands). In addition, inflammation and edema are less definitive features than stromal desmoplasia as these can be seen with AIS. In some cases, small foci with the earliest suggestion of architectural aberrations, including gland branching, gland fusion, and interglandular cribriform growth, can raise concern for early stromal invasion but it may not be possible to unequivocally render a definitive diagnosis of invasion or achieve a consensus interpretation when reviewing with colleagues (Figure 3). In such situations, a less definitive diagnosis indicating concern for foci of early stromal invasion in the setting of extensive AIS may need to be rendered (see below for reporting recommendation). One reason for potentially adopting a
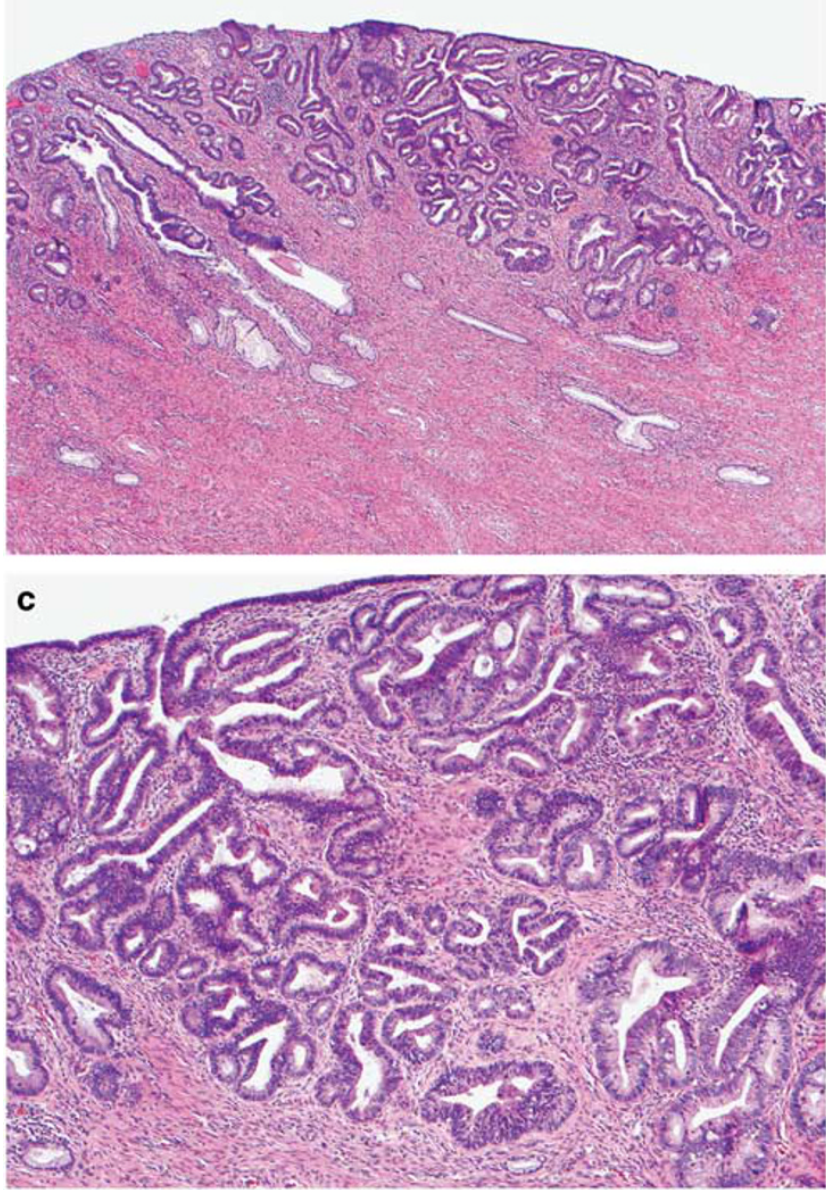

b
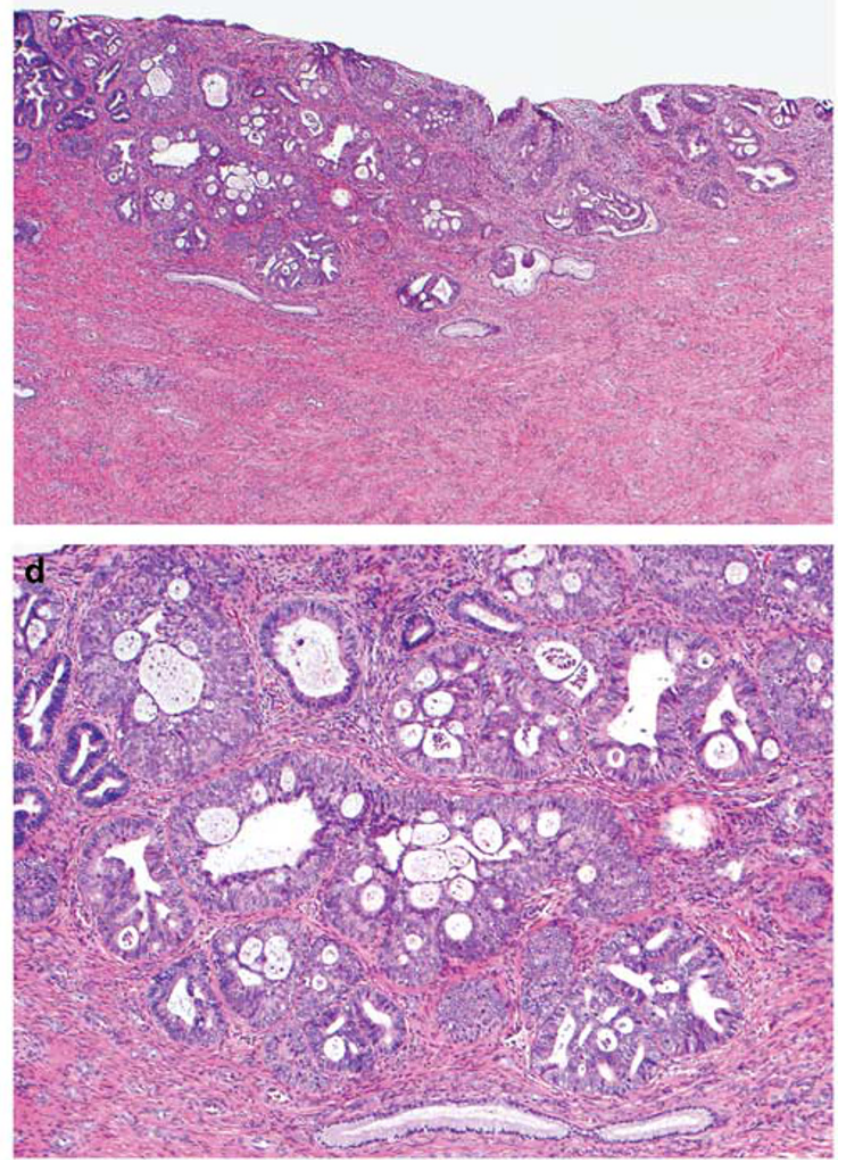

Figure 3 Extensive AIS with small foci concerning for early/subtle stromal invasion. The lesion is long and thin and doesn't extend beyond the level of normal endocervical glands (a and $\mathbf{b})$. Small foci with early gland branching/fusion and focal interglandular cribriform growth favor early invasive adenocarcinoma (c and $\mathbf{d})$.

Figure 2 Endocervical adenocarcinomas comprised of extensive adenocarcinoma in situ (AIS) intimately admixed with invasive components having deceptive patterns of invasion ('AIS-like'). A very superficial lesion has a subtle stromal reaction and some suggestion of early gland branching (a-c). An extensive superficial proliferation has tightly clustered glands and early gland fusion, with some surrounding stromal desmoplasia (d-f). A surface/exophytic villoglandular proliferation is more extensive than allowed in AIS and has focal invasion of underlying stroma by 'AIS-like' glands with a surrounding stromal reaction (g-i). An extensive, haphazard proliferation extends deeply into cervical stroma and displays some focal early gland fusion but lacks stromal desmoplasia (j-l). These four examples were all associated with high-risk HPV-positive ovarian metastases. 'AIS-like' glands extend linearly into deeper cervical stroma in close proximity to thick-walled blood vessels but there is no stromal reaction $(\mathbf{m}-\mathbf{o})$. 
a more conservative approach to diagnosing such cases is to avoid more aggressive surgical staging for tumors that appear to have little risk of lymph node metastases (see discussion below on the pattern-based classification system). However, occasional cervical tumors with limited invasion, including some for which a diagnosis of AIS was rendered on original review and in some instances also in consultation, have had ovarian metastases. ${ }^{9}$ In some of these cases, the cervical lesions have been long and thin, at times extending into the lower uterine segment, such that the dimensions of the entire lesion (in situ and invasive foci combined) are greater than that allowed for conventional 'microinvasive carcinoma' even if size of the invasive foci appear to conform to the required dimensions for 'microinvasion'. This has led to speculation that the cervical neoplasms may spread trans-tubally to the ovary. These ovarian tumors are often misclassified as independent primary neoplasms, usually as mucinous/endometrioid borderline/atypical proliferative tumors, with or without intraepithelial carcinoma. Although some of these patients present with concurrent cervical and bilateral ovarian tumors, making recognition of the ovarian tumors as metastases easier, the ovarian tumors can present prior to or some time (even years) after the cervical tumor and can be large, multicystic, and unilateral, with 'borderline-like' growth patterns which make it challenging to recognize these tumors as metastatic or even invasive. The difficulty in recognizing the cervical proliferations as invasive rather than extensive AIS contributes to the potential for misclassification of the ovarian tumors as independent primary neoplasms. Such cases are often submitted for consultation to address the relationship of the cervical and ovarian tumors, demonstrating the challenge posed by these tumors and emphasizing the need for pathologists to be aware of this scenario. The presence of identical high-risk HPV types in the cervical and ovarian tumors in all paired tumors analyzed supports interpretation of the ovarian tumors as metastases (ref. 9 and author's experience). Experience with these cases has led to lowering of the threshold for suspecting and diagnosing subtle/deceptive 'AIS-like' patterns of invasion in the setting of extensive AIS, such that some lesions are much more readily and unequivocally diagnosed as invasive now than some years ago, not only in consultation but also in routine practice. However, there needs to be a balance between lowering the threshold for diagnosing invasion and avoiding unnecessarily aggressive surgical staging.

Recommendations for reporting (preferred diagnostic terminology) and measuring these tumors, particularly those with 'AIS-like' patterns of invasion include the following:

1. The term 'in situ (AIS) and invasive endocervical adenocarcinoma (usual type)' is recommended for high-risk HPV-related tumors.

2. The term 'microinvasive carcinoma' should be avoided because this term is no longer recommended for squamous neoplasms and is not well-defined for glandular neoplasms due to measuring difficulties (see point 3); of note, the term 'superficially invasive carcinoma' is reserved for squamous neoplasms per the College of American Pathologists (CAP) Lower Anogenital Squamous Terminology (LAST) recommendations. ${ }^{10}$

3. When invasion is present but AIS and the invasive component are intimately associated and cannot be reliably distinguished, the dimensions of the entire lesion should be measured. This includes the thickness (usually measured from the surface), which replaces the depth parameter and the horizontal/lateral extent; a

Table 1 Reporting suggestions for challenging endocervical glandular proliferations

Pathologic situation Suggested reporting language

Lesions for which AIS and 'AIS-like' patterns of invasion are intimately associated

Lesions extending to the margin of an excisional specimen

Lesions assessed as equivocal for invasion
'The in situ and invasive components are sufficiently intimately associated that they cannot be clearly separated for the purpose of measuring the dimensions of only the invasive component. Therefore, the dimensions of the entire lesion are provided. The entire lesion (in situ and invasive components combined) measures _ $\mathrm{mm}$ in thickness and _ $\mathrm{mm}$ in horizontal extent.'

'Since the lesion extends to the margin, the possibility of residual/additional lesion in remaining cervical tissue cannot be excluded. Therefore, the size/extent of the invasive component in this specimen cannot be guaranteed to represent the entire invasive lesion.'

'Some of the lesional foci have features suggesting the possibility of a subtle 'AIS-like' pattern of superficial stroma invasion. However, because criteria for reproducibly diagnosing such subtle patterns of early/superficial stromal invasion have not been established, and since there is overlap with the patterns allowed in AIS, these foci are concerning but are not interpreted as unequivocally diagnostic of invasion.' 

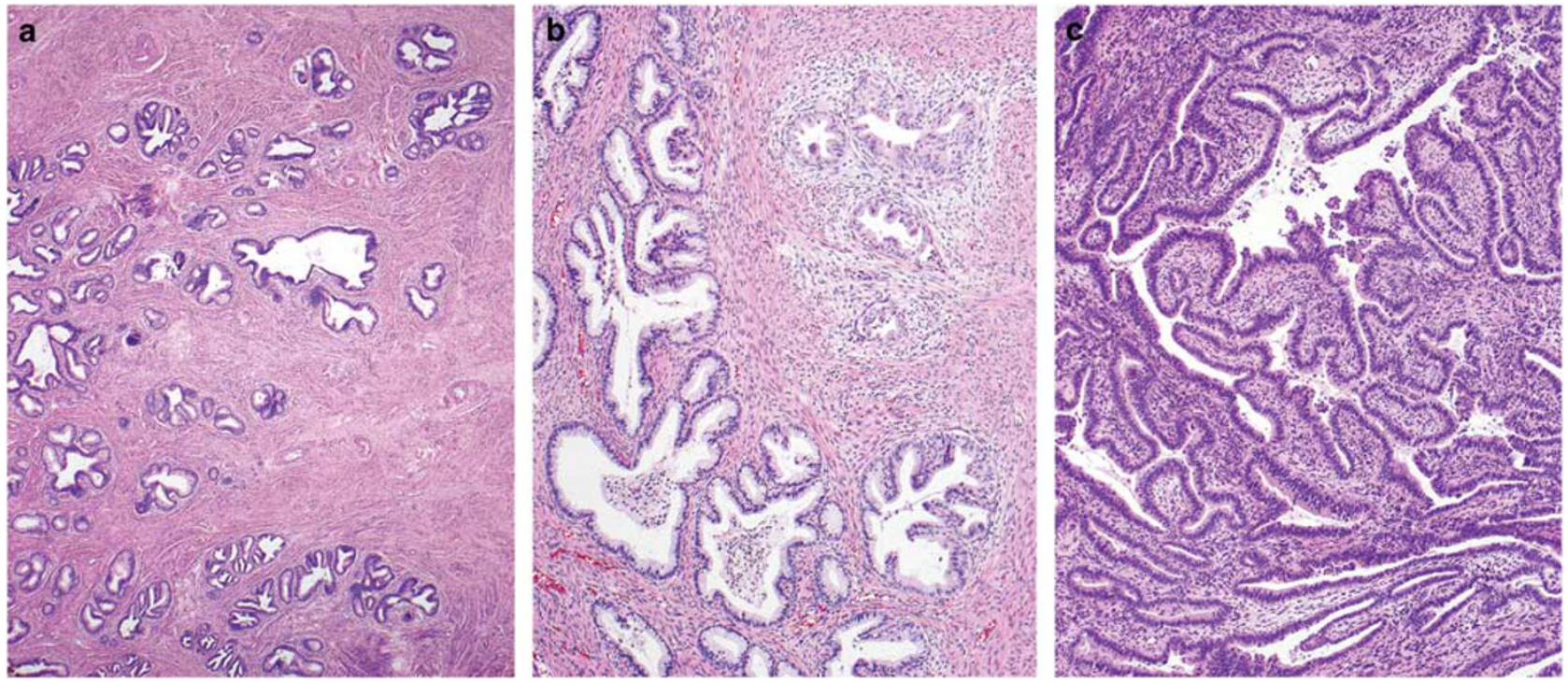

Figure 4 Pattern-based classification system for invasive endocervical adenocarcinoma. Pattern A: Well-demarcated glands with 'AIS-like' growth pattern, without stromal desmoplasia (a). Pattern B: Focal destructive invasion arising from well-demarcated (pattern A) glands (b). Pattern C: Diffuse destructive invasion by branching interconnected glands with stromal desmoplasia (c).

third dimension can be calculated if desired (number of blocks with tumor multiplied by the tissue slice thickness). Suggested language for reporting problematic situations is provided in Table 1.

A recently proposed pattern-based classification system recommends use of patterns of invasion rather than traditional measurement of depth of invasion and horizontal extent to better assess risk of lymph node metastases (Figure 4). ${ }^{11}$ Three patterns are recognized in this system:

1. Pattern A (Figure 4a), defined by the following features/criteria: well or moderately differentiated adenocarcinoma composed of well-demarcated glands lacking a desmoplastic stromal reaction and confluent growth (no greater than a $4 \times$ field) but with complex intraglandular growth allowed (cribriform, papillary), no single invasive cells, and no lymph-vascular invasion; depth of invasion and relationship to large vessels are irrelevant. This pattern was not associated with lymph node metastases. While tumors with such 'AIS-like' patterns may well have little or no risk of lymph node metastases, ovarian metastases do occur, as discussed above.

2. Pattern B (Figure 4b), characterized by early destructive stromal invasion arising from well-demarcated glands (pattern A glands), with or without lymph-vascular invasion. The risk of lymph node metastases for these tumors was $4.4 \%$.

3. Pattern C (Figure 4c), characterized by diffuse destructive invasion. The risk of lymph node metastases for these tumors was $23.8 \%$.

\section{Distinguishing high-risk HPV-related (usual type) endocervical adenocarcinomas from endometrial endometrioid carcinomas and related subtypes (those with mucinous and metaplastic-type differentiation)}

There are a number of reasons why distinction of high-risk HPV-related (usual type) endocervical adenocarcinomas from endometrial endometrioid carcinomas and their related subtypes (those with mucinous and metaplastic-type differentiation) can be problematic. These include:

1. Shared cellular differentiation. Both tumor types can have mucinous and endometrioid-like features. High-risk HPV-related endocervical adenocarcinoma is most often characterized by a hybrid of mucinous and endometrioid-like features, often with numerous apically situated mitotic figures and basally situated apoptotic bodies (Figure 5a). Varying degrees of mucinous differentiation are often present, with some dominated by mucinous features (Figure $5 \mathrm{~b}$ ) and, less commonly, some displaying endometrioidlike features (Figure 5c). Most endometrial carcinomas are of the endometrioid type but some can have varying degrees of mucinous differentiation.

2. Shared architecture. Tumors of both types most often are entirely well differentiated glandular proliferations and both can have villoglandular growth patterns (Figure $5 \mathrm{~d}$ ).

3. Involvement of both the endometrial and endocervical components of a fractional curettage specimen. 

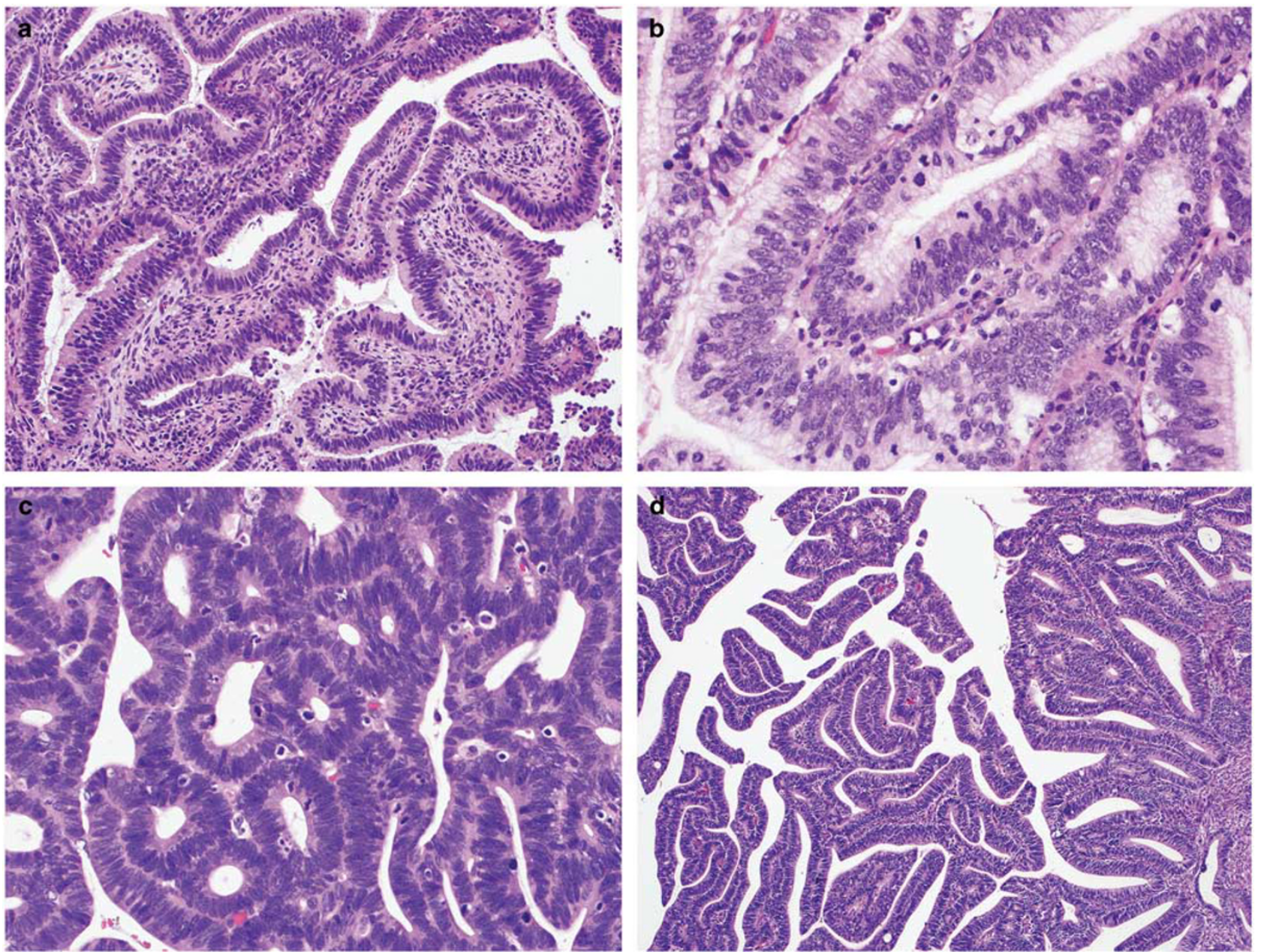

Figure 5 High-risk HPV-related endocervical adenocarcinomas. Common hybrid of mucinous and endometrioid features (usual type), with numerous mitotic figures and apoptotic bodies (a). Tumor with more evident mucinous features (b). Tumor with distinct endometrioid features, simulating a primary endometrial endometrioid carcinoma (c). Tumor with villoglandular pattern, which can be shared by endocervical and endometrial endometrioid carcinomas (d).

4. Lack of precursor lesions. This is often a problem in biopsy and curettage specimens but can also be problematic in hysterectomy specimens when tumors involve both the endometrium (often also intervening lower uterine segment) and the endocervix. In this situation, precursor lesions can be either overgrown by carcinoma or can simulate one another (eg endocervical adenocarcinoma extending into endometrium simulates complex atypical hyperplasia; Figure 6).

5. The dominant tumor component is not necessarily the primary site. Some endocervical adenocarcinomas can have limited endocervical involvement and dominant endometrial or endomyometrial involvement, simulating a primary endometrial adenocarcinoma with endocervical extension (Figure 6). ${ }^{12}$

In routine practice, a few selected immunohistochemical markers can readily distinguish most tumors (Table 2). The most useful immunohistochemical markers are p16 and hormone receptors (estrogen and progesterone receptors (ER/PR)), with p16 as the most useful single marker followed by PR and ER. ${ }^{13,14}$ High-risk HPV-related endocervical adenocarcinomas exhibit diffuse/moderate-strong p16 expression (essentially all tumor cells are positive) which serves as a surrogate marker for high-risk HPV in this setting (Figure 7a). High-risk HPV detection within tumor tissue by in situ hybridization (Figure 7b) is definitive for identification of this group of endocervical adenocarcinomas; however, DNA in situ hybridization assays are not $100 \%$ sensitive. ${ }^{13,14}$ High-risk HPV-related endocervical adenocarcinomas typically lose hormone receptor expression (Figures $7 \mathrm{c}$ and d). ${ }^{15}$ Some have retained ER expression (sometimes diminished/ weaker and patchy/focal compared with the typically strong expression in normal glands) with loss of PR expression (Figures 7e and f), and some retain significant expression of both ER and PR; thus, PR is 

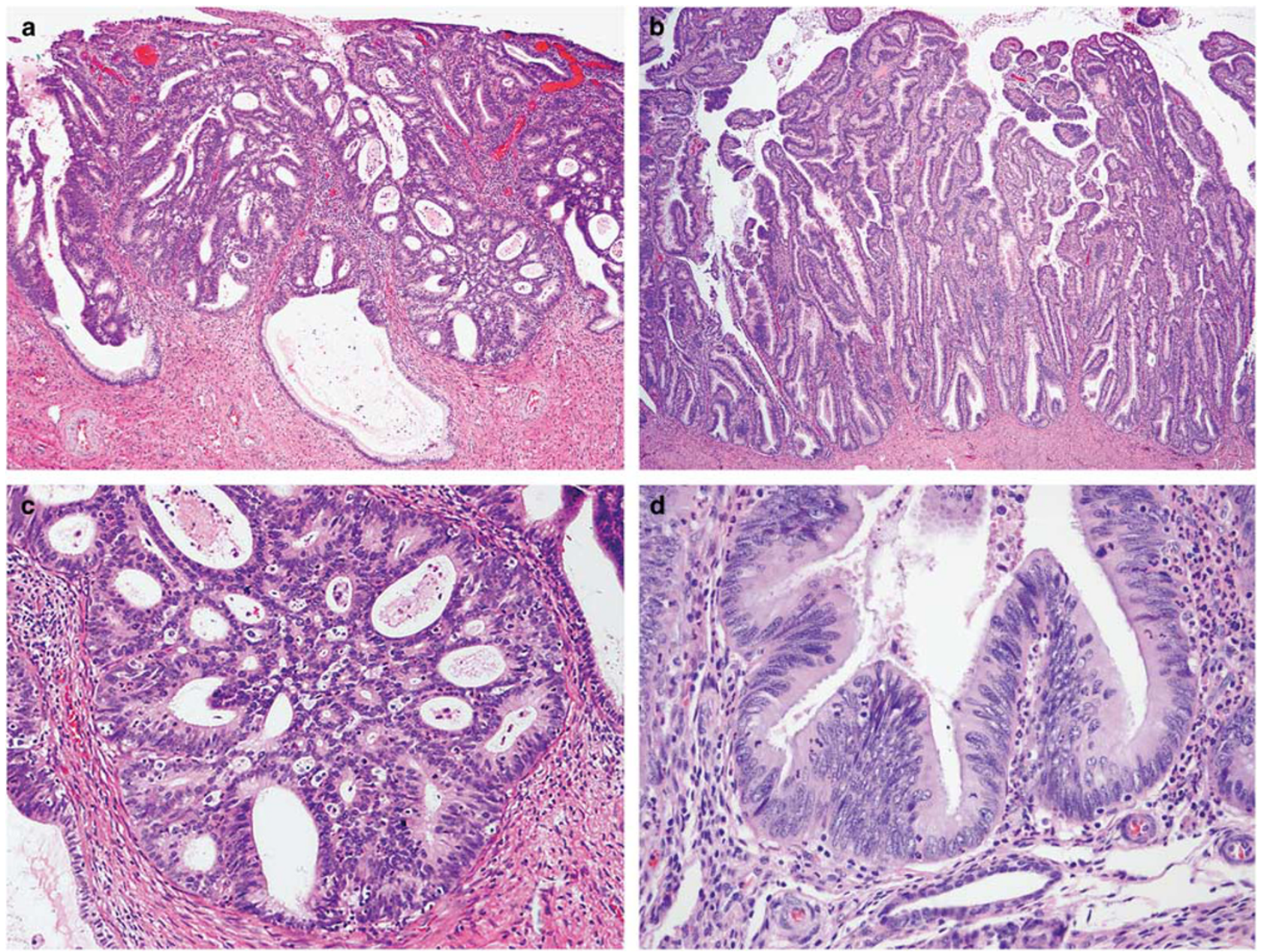

Figure 6 Endocervical adenocarcinoma with extensive endometrial involvement. Tumor in endocervix is superficial and not the dominant component (a). Endometrial component simulates a primary endometrial endometrioid carcinoma (with associated complex atypical hyperplasia) with endocervical extension (b). Cytologic features in both the endocervical (c) and endometrial (d) components, manifested as a hybrid of endometrioid and mucinous features with evident mitotic figures and apoptotic bodies, favor a high-risk HPV-related endocervical adenocarcinoma. Ancillary studies confirmed the tumor as high-risk HPV-related endocervical adenocarcinoma (tumor was diffusely positive for p16, negative for hormone receptors, and contained high-risk HPV DNA by in situ hybridization (data not shown; see Figure 7 for examples)).

more discriminatory than ER (ref. 9 and author's experience). In contrast, endometrial endometrioid carcinomas of all grades display variable patchy p16 expression ranging from limited to extensive, with the more positive tumors still usually having $<80 \%$ of the tumor cells positive (non-diffuse pattern), with scattered negative or weak foci/patches or interspersed individual negative tumor cells present (Figures 8a-f). ${ }^{13,14}$ Some tumors with prominent mucinous/metaplastic-type differentiation can have more extensive p16 expression but the staining intensity is usually not as strong as is seen with high-risk HPV-related endocervical adenocarcinomas and some negative patches are usually present if the sample is not too small (Figures 8g and h; ref. 9 and author's experience). Most endometrial endometrioid carcinomas express hormone receptors (both ER and PR; Figures $8 \mathrm{i}$ and j). ${ }^{15}$ Of note, immunohistochemical analysis of p16 and hormone receptor expression is useful only for distinction of high-risk HPV-related endocervical adenocarcinomas from endometrial endometrioid carcinomas. Serous carcinomas virtually always have diffuse/strong p16 expression and typically lose hormone receptor expression (ER-/PR-) so they share this immunoprofile with high-risk HPV-related endocervical adenocarcinomas. ${ }^{14}$ High-risk HPV detection definitively identifies the latter but if not readily available or when insensitive, immunohistochemical analysis of p53 expression is useful for distinction of these tumors. Serous carcinomas virtually always have either diffuse p53 overexpression or are completely negative (so-called 'null' pattern; with both patterns being highly correlated with p53 mutation), whereas high-risk HPV-related endocervical adenocarcinomas have 
Table 2 Biomarker profile of uterine adenocarcinomas

\begin{tabular}{lllll}
\hline Marker & $\begin{array}{l}\text { Endocervical } \\
\text { adenocarcinoma, } \\
\text { usual type }\end{array}$ & $\begin{array}{l}\text { Endocervical } \\
\text { adenocarcinoma, } \\
\text { gastric type }\end{array}$ & $\begin{array}{l}\text { Endometrial } \\
\text { endometrioid } \\
\text { carcinoma }\end{array}$ & Serous carcinoma \\
\hline p16 & Positive (diffuse) & Negative or patchy positive & $\begin{array}{l}\text { Variably positive } \\
\text { (patchy/non-diffuse) } \\
\text { Usually no significant } \\
\text { expression }\end{array}$ & $\begin{array}{l}\text { Positive (diffuse) } \\
\text { Positive (diffuse) or completely } \\
\text { negative } \\
\text { ER }\end{array}$ \\
No significant expression & Some positive (diffuse) & Positive & Negative \\
PR & Negative $^{\mathrm{d}}$ & Negative & Negative & \\
High-risk HPV & $\begin{array}{l}\text { Negative } \\
\text { Positive }\end{array}$ & Negative & Negative & Negative \\
\hline
\end{tabular}

${ }^{a}$ Varies from limited to extensive expression but usually not diffuse (few exceptions). ${ }^{b}$ Limited expression, usually scattered cells with weak-moderate expression, referred to as 'wild-type' pattern-neither diffuse nor completely negative. ' Usually limited expression, most commonly scattered cells with weak-moderate expression, referred to as 'wild-type' pattern but some higher-grade tumors can have diffuse expression. ${ }^{\mathrm{A}} \mathrm{A}$ minority retains expression, usually ER $>$ PR (some ER+/PR - , few ER+/PR+).

limited/patchy weaker p53 expression (so-called 'wild-type' pattern). Most endometrial endometrioid carcinomas share this limited/patchy ('wild-type') p53 expression pattern with endocervical adenocarcinomas but some higher-grade examples of the former can have diffuse p53 over-expression. The immunoprofile of endocervical adenocarcinomas of the type unrelated to high-risk HPV is discussed in the next section. The traditional immunohistochemical approach using CEA and vimentin, which is based on the expectation that endometrial endometrioid carcinoma is vimentin-positive and CEA-negative and that endocervical adenocarcinoma is vimentin-negative and CEA-positive, is problematic for several reasons: (1) expression of these markers can be focal; (2) staining can be difficult to interpret because CEA can be only apical/ glycocalyceal or also cytoplasmic and it can be difficult to determine whether vimentin expression is actually within glands versus in closely apposed stroma, and (3) tumors with mucinous differentiation usually express CEA, regardless of origin. Of note, CD10 has no role in determining location/origin of tumor because expression is not specific for endometrial stroma (endocervical stroma can be positive).

\section{Distinguishing benign mucinous endocervical glandular proliferations, including the various forms of endocervical glandular hyperplasia, from gastric-type mucinous endocervical adenocarcinomas, particularly the very well differentiated minimal deviation type}

There are 2 specific forms of endocervical glandular hyperplasia, namely, diffuse laminar endocervical glandular hyperplasia (Figures 9a-c) and lobular endocervical glandular hyperplasia (Figures 9d-f); the latter has an uncommon atypical form (see below). In addition, there are examples which are probably best regarded as not otherwise specified because they lack classical complete features of one of the above processes; these likely represent examples of florid tunnel clusters (Figures 9g-i). In addition, dilated and elongated endocervical glands can at times extend below the usually expected level in the cervical wall, raising concern for a malignant neoplasm. These benign and hyperplastic lesions must be distinguished from well differentiated gastric-type mucinous adenocarcinomas, which include minimal deviation adenocarcinoma (adenoma malignum) as the most highly differentiated form. General features of benign/hyperplastic mucinous endocervical glandular proliferations, which are more commonly encountered than the adenocarcinomas, include:

1. Organized lobular or diffuse patterns, confined to the inner $1 / 3-1 / 2$ of the cervical wall and sharply demarcated from underlying stroma.

2. Lack of stromal desmoplasia (inflammation and focal stromal edema can be present).

3. Bland to mildly atypical (reactive) nuclear features.

4. No mitotic activity.

Diffuse laminar endocervical glandular hyperplasia is an incidental finding in a hysterectomy specimen characterized by the following features (Figures 9a-c): ${ }^{16}$

1. Location confined to the inner $1 / 3$ of the cervical wall.

2. A laminar proliferation of closely packed glands sharply demarcated from underlying stroma.

3. Diffuse to vaguely lobular architecture.

4. Rounded glands which can occasionally be irregular and star-shaped.

5. Tall columnar mucinous epithelium.

6 . Bland basally located nuclei, occasionally with focal reactive changes.

7. No appreciable mitotic activity. 

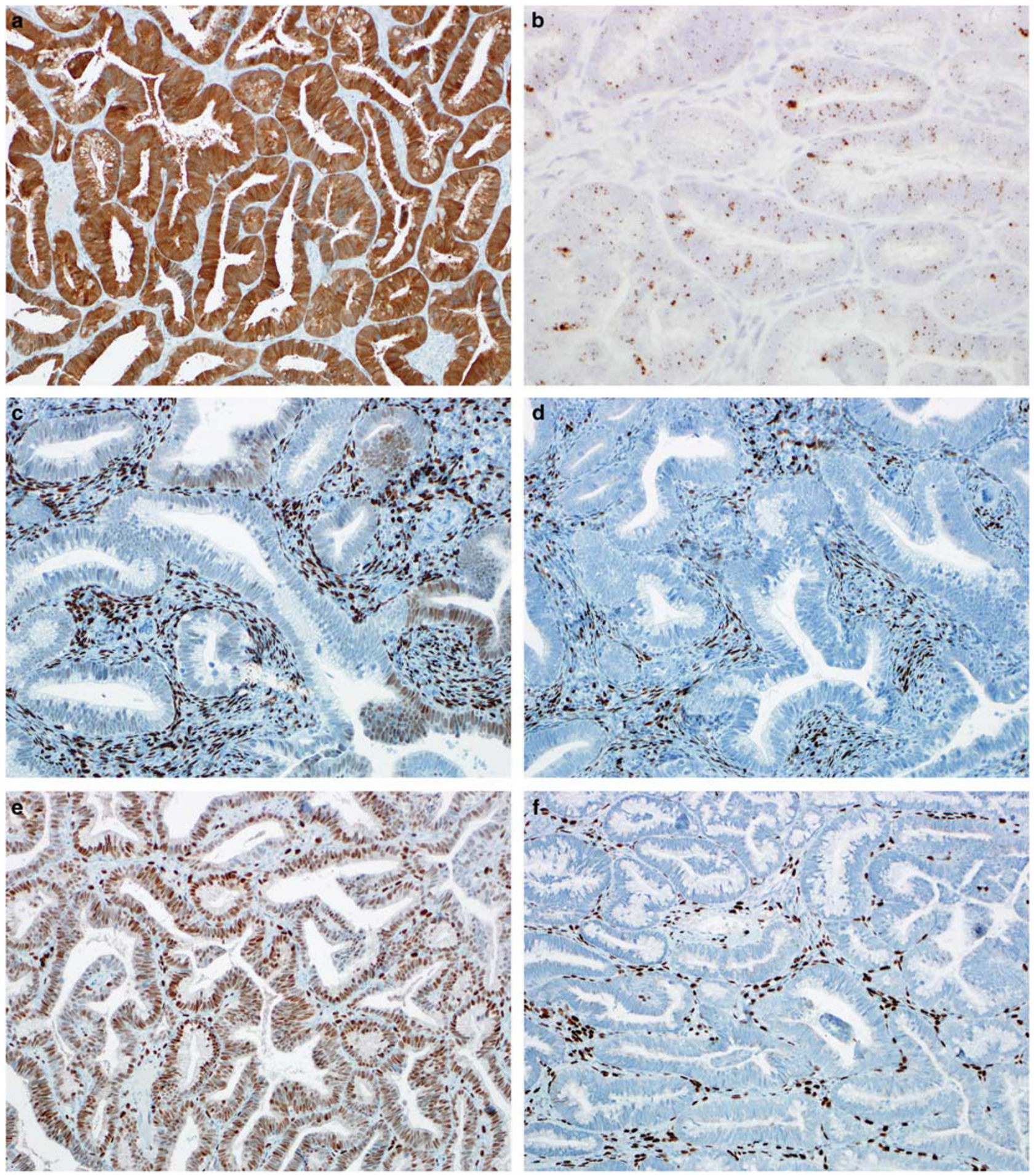

Figure 7 Characteristic immunoprofile of high-risk HPV-related (usual type) endocervical adenocarcinomas. Tumor displays diffuse/ strong p16 expression (a). High-risk HPV DNA is detected by in situ hybridization (b (punctate nuclear signals)), consistent with the p16 expression pattern. Tumor is essentially negative for ER (c (rare positive patches are present)) and PR (d). Another example has retained expression of ER (e) with loss of expression of PR (f).

8. An inflammatory infiltrate, occasionally with focal stromal edema and disrupted glands with extruded mucin.
Lobular endocervical glandular hyperplasia is usually an incidental finding but can present as a visible grossly well-demarcated mass or cystic 
lesion, or with clinical symptoms (watery or mucoid discharge). It is characterized by the following features (Figures 9d-f): ${ }^{17,18}$

1. Location confined to the inner $1 / 2$ of the cervical wall.
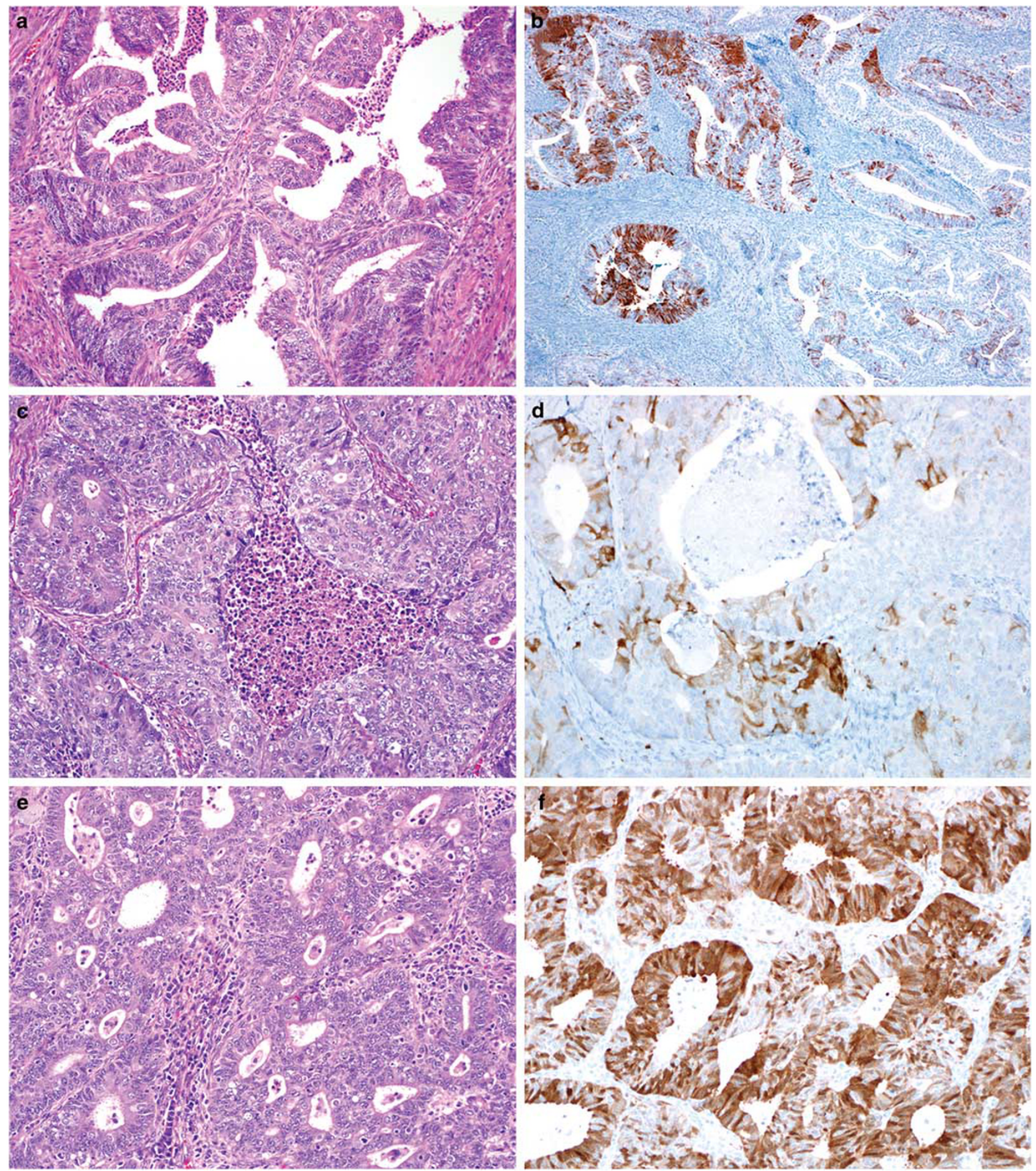

Figure 8 Characteristic immunoprofile of endometrial endometrioid carcinomas. Patchy (non-diffuse) p16 expression in a FIGO grade 1 tumor (a and b). Patchy (non-diffuse) p16 expression in a FIGO grade 3 tumor (c and $\mathbf{d}$ ). Extensive yet non-diffuse p16 expression (negative interspersed cells are present) in a FIGO grade 1 tumor (e and f). Extensive yet non-diffuse p16 expression (negative/weak patches are present) in a FIGO grade 1 tumor with extensive metaplastic-type differentiation ( $g$ and $\mathbf{h}$ ). Extensive expression of ER (i) and PR (j). 

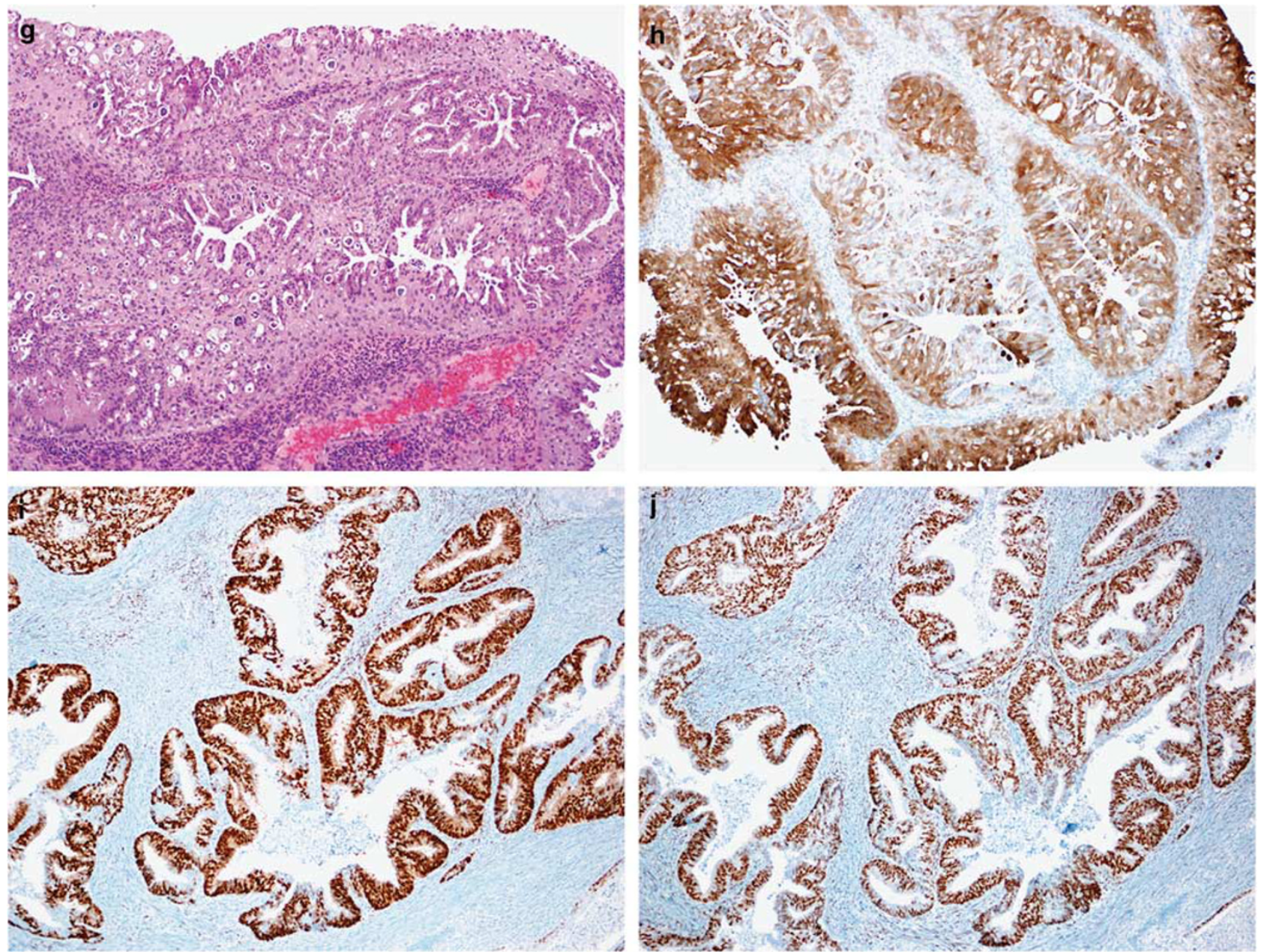

Figure 8 Continued.

4. No significant cytologic atypia or mitotic activity.

5. A gastric/pyloric immunophenotype (MUC6+, HIK1083+).

6. Loss of hormone receptor expression (ER-/PR-).

Gastric-type endocervical adenocarcinomas are unrelated to high-risk HPV. They display immunohistochemical evidence of gastric-type/pyloric differentiation (MUC6+, HIK1083+) but the morphologic features are sufficiently distinctive that immunohistochemical analysis is not necessary in most cases. These adenocarcinomas are characterized by glands lined by cells with abundant pale to eosinophilic cytoplasm and distinct cell borders, with evident malignant cytologic features (vesicular nuclei often with prominent nucleoli), and vary from well to poorly differentiated (Figures 10a-d). ${ }^{3,6,7,19}$ Minimal deviation adenocarcinomas (adenoma malignum) represent the highly differentiated form of this tumor. ${ }^{20}$ They are characterized by numerous deceptively bland glands, which often lack surrounding stromal desmoplasia. However, the very well differentiated glands typically exhibit deep invasion, are haphazardly distributed, and display architectural abnormalities, including intraglandular papillary protrusions and irregular profiles. At least focally, some glands display malignant cytologic features (vesicular nuclei with distinct/prominent red nucleoli) and are associated with stromal desmoplasia features, which enable recognition as invasive adenocarcinoma (Figures 10e-k). In contrast to high-risk HPV-related endocervical adenocarcinomas, p16 is typically negative or focally positive (Figure 10l). P53 is sometimes diffusely positive (Figure 10m). However, gastric-type adenocarcinomas share loss of hormone receptor expression (ER-/PR-) with high-risk HPV-related endocervical adenocarcinomas (Figures 10n and o). These adenocarcinomas are characterized by aggressive clinical behavior. When evaluating mucinous endocervical glandular proliferations, the features that raise concern for gastric-type well differentiated/ minimal deviation adenocarcinoma include the following:

1. A haphazard/disorganized proliferation (glands having the appearance of being 'strewn across the 
slide') which is typically extensive but not necessarily as densely packed as hyperplasia.

2. Architecturally abnormal glands, including branching and papillary infoldings.

3. Deep extension into the cervical wall.

4. Close proximity to thick-walled vessels.

5. Distinctive nuclear atypia characterized by vesicular chromatin with discrete red nucleoli.

6. Cytologically malignant glands associated with stromal desmoplasia.
An atypical form of lobular endocervical glandular hyperplasia has been recognized as a potential precursor of gastric-type/minimal deviation adenocarcinoma. ${ }^{19}$ It has atypical architectural and cytologic features (Figures 11a-d), including:

1. Infolding of epithelium or papillary projections with fine fibrovascular stroma.

2. Nuclear enlargement.

3. Irregular nuclear contour.
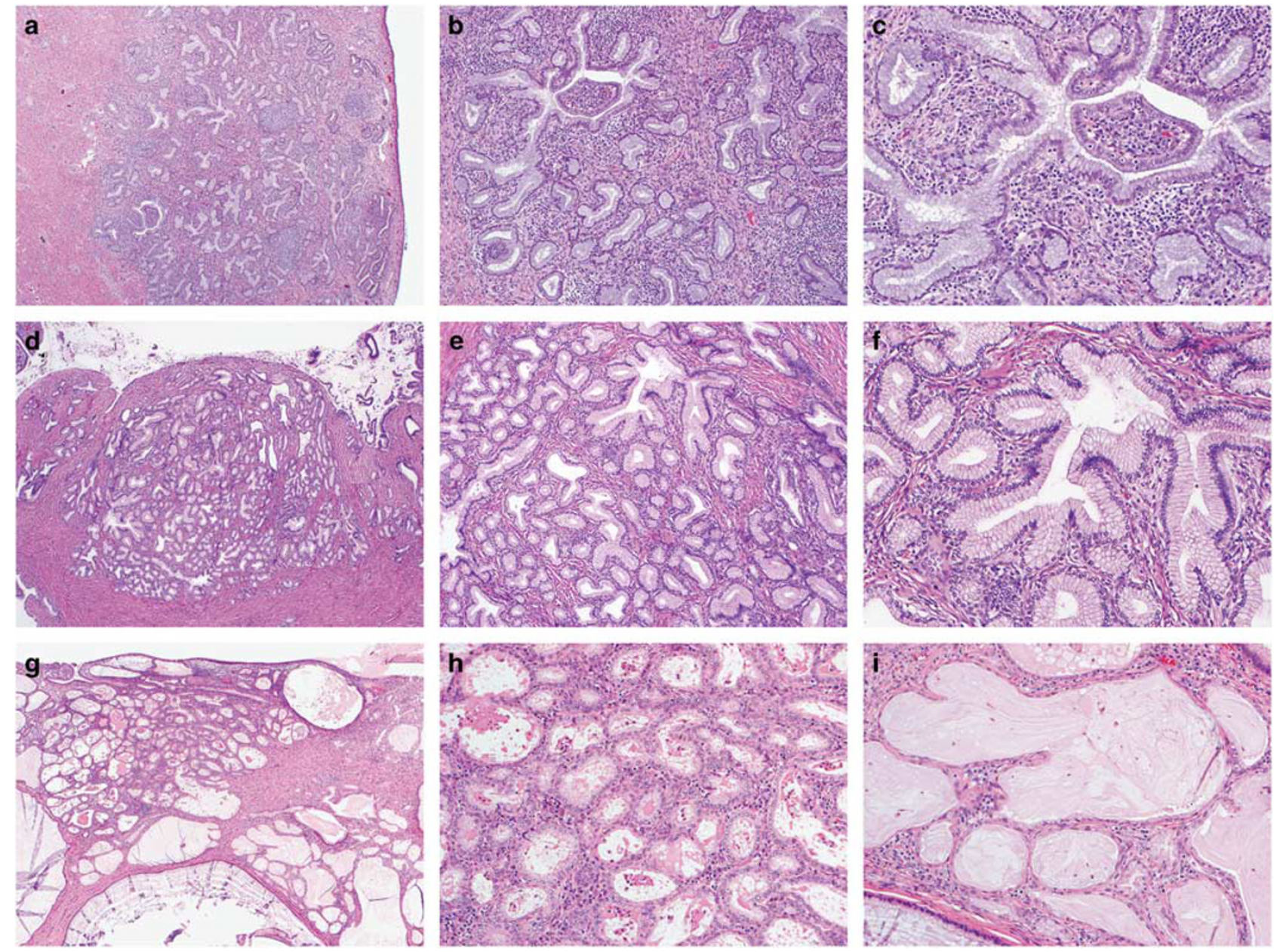

Figure 9 Endocervical glandular hyperplasias. Diffuse laminar endocervical glandular hyperplasia is characterized by a crowded proliferation which is sharply demarcated from the underlying stroma and composed of bland mucinous glands with surrounding inflammation (a-c). Lobular endocervical glandular hyperplasia is characterized by a crowded lobulated proliferation which is sharply demarcated from the underlying stroma and composed of bland mucinous glands with pyloric-type features (d-f). Endocervical glandular hyperplasia, not otherwise specified, is characterized by aggregates of crowded glands with a combination of rounded and dilated/ attenuated bland mucinous glands, likely representing coalescence of florid tunnel clusters (g-i).

Figure 10 Gastric-type endocervical adenocarcinomas. Tumors are characterized by well-differentiated mucinous glands with pale to eosinophilic cytoplasm, distinct cell borders, and distinct nuclear atypia (vesicular nuclei with discrete nucleoli) (a-c). Tumors can have poorly differentiated components (d). Minimal deviation adenocarcinomas (e-k), representing the most differentiated form of gastric-type adenocarcinoma, have a diffuse, haphazard ('glands strewn across the slide' appearance), and deeply extending proliferation (e-g) of very well differentiated mucinous glands with some architectural abnormalities (h), varying from deceptively bland (i) to cytologically malignant (j and $\mathbf{k})$, with many lacking surrounding stromal desmoplasia but some having associated desmoplasia (k). The characteristic immunoprofile is p16 negative to focally positive (l), with diffuse p53 expression in some cases (m), and loss of expression of ER (n) and PR (o). 

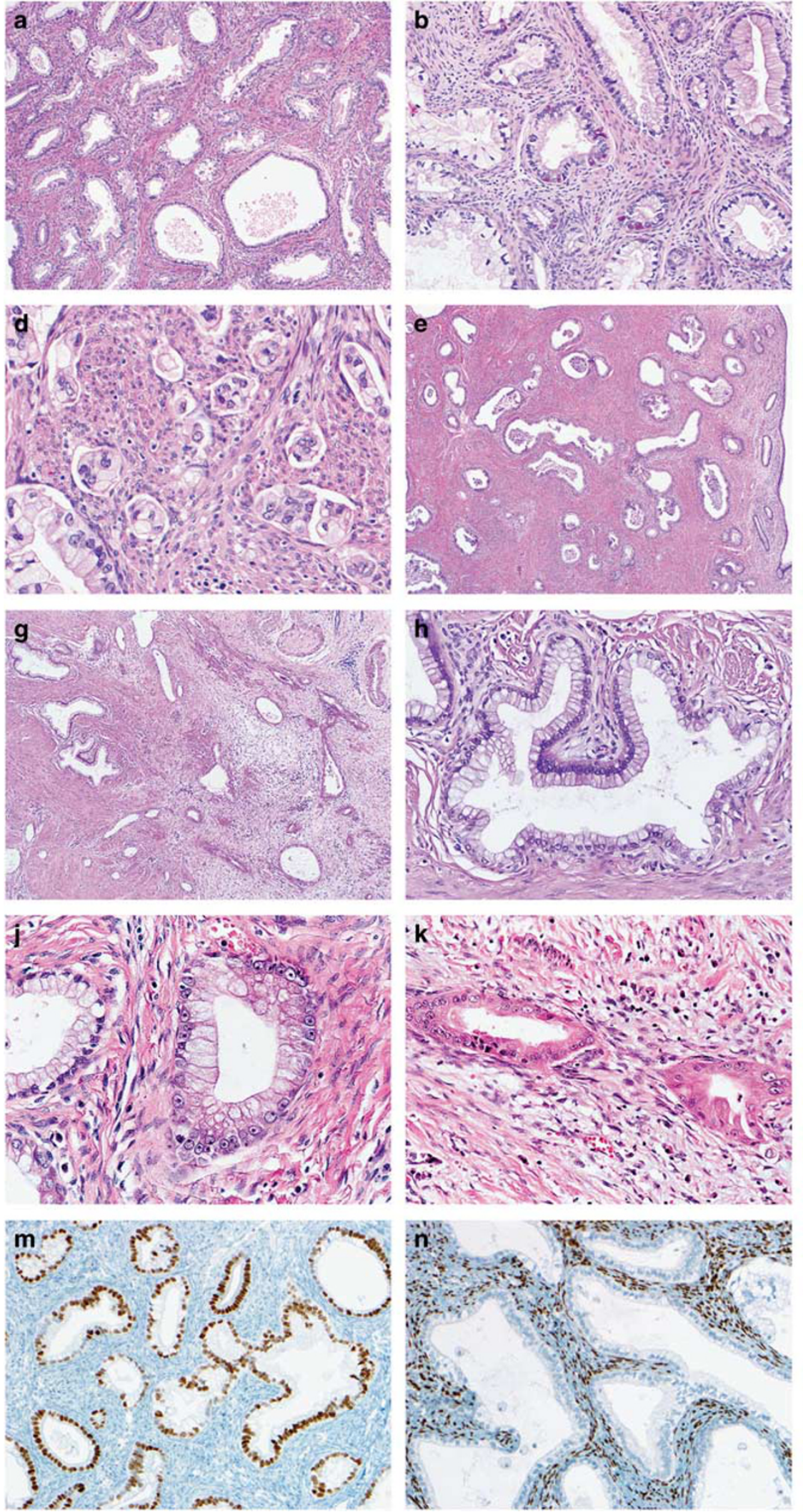
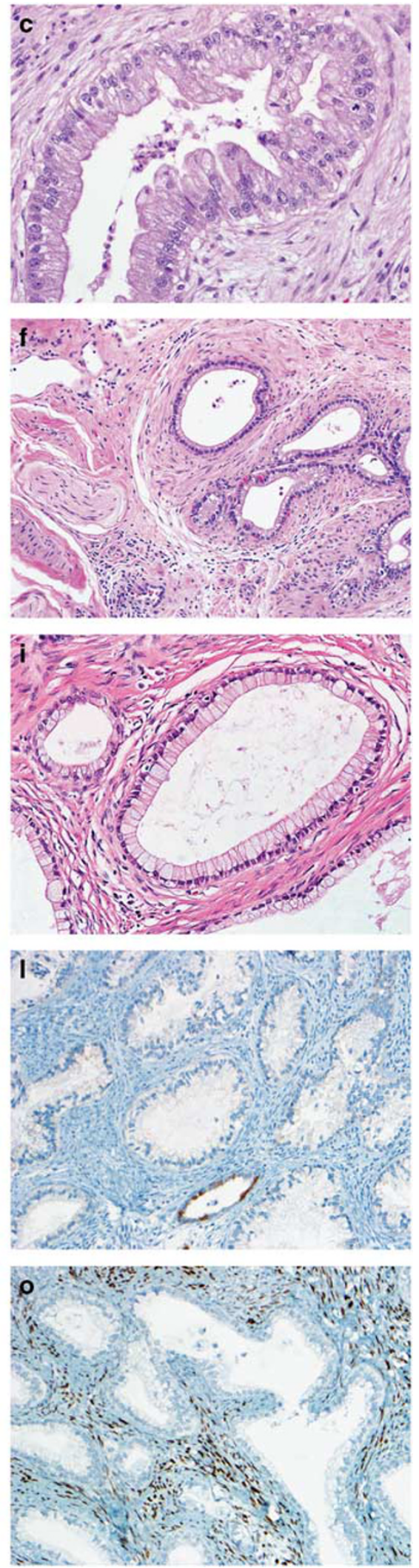

Figure 10 For caption see page S25. 

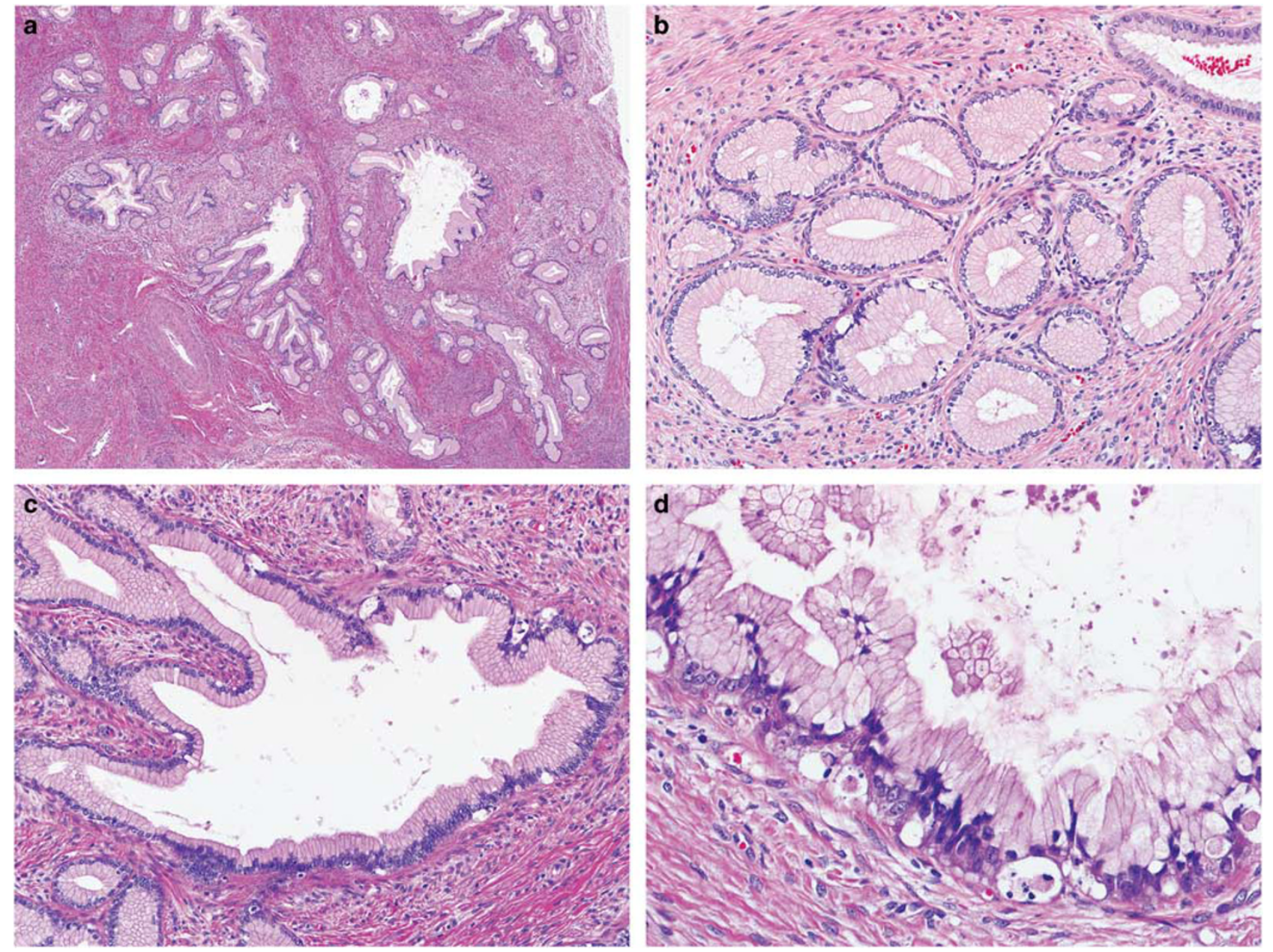

Figure 11 Atypical lobular endocervical glandular hyperplasia (associated with gastric-type adenocarcinoma). A lobulated to diffuse proliferation of well differentiated mucinous glands with some architectural abnormalities, close apposition to a thick-walled vessel, and some stromal desmoplasia suggesting a transition from lobular endocervical glandular hyperplasia to well differentiated gastric-type/ minimal deviation adenocarcinoma (a); definitive deeply invasive gastric-type adenocarcinoma was evident elsewhere (not shown). Some areas have features of typical lobular endocervical glandular hyperplasia (b, rounded pyloric-type glands) but other glands have architectural and cytologic abnormalities, including intraglandular papillary projections (c) and epithelial tufting (d), with some nuclear atypia and numerous apoptotic bodies (d).

4. Distinct nucleoli.

5. Coarse chromatin texture.

6. Loss of polarity.

7. Occasional mitotic figures.

8. Apoptotic bodies and/or nuclear debris in the lumen.

Endocervical adenocarcinomas continue to pose diagnostic challenges in routine practice. Additional studies are needed to determine whether novel approaches, such as new classification systems for assessing invasion, can provide clinically relevant and reproducible methods for evaluating invasive endocervical adenocarcinomas. It would also be of great value to identify novel biomarkers to assist in distinguishing in situ from invasive endocervical adenocarcinomas and benign from malignant mucinous endocervical glandular proliferations.

\section{Disclosure/conflict of interest}

The author declares no conflict of interest.

\section{References}

1 Wilbur D, Colgan T, Ferenczy A et al. Tumours of the uterine cervix: Epithelial tumours - Glandular tumours and precursors. In: Kurman RJ, Carcangiu ML, Herrington S, Young RH (eds). World Health Organization Classification of Tumours of Female Reproductive Organs. 4th (edn). IARC Press: Lyon, France, 2014.

2 Young RH, Clement PB. Endocervical adenocarcinoma and its variants: their morphology and differential diagnosis. Histopathology 2002;41:185-207.

3 McCluggage WG. New developments in endocervical glandular lesions. Histopathology 2013;62:138-160. 
4 Pirog EC, Kleter B, Olgac S et al. Prevalence of human papillomavirus DNA in different histological subtypes of cervical adenocarcinoma. Am J Pathol 2000;157: 1055-1062.

5 An HJ, Kim KR, Kim IS et al. Prevalence of human papillomavirus DNA in various histological subtypes of cervical adenocarcinoma: a population-based study. Mod Pathol 2005;18:528-534.

6 Mikami Y, McCluggage WG. Endocervical glandular lesions exhibiting gastric differentiation: an emerging spectrum of benign, premalignant, and malignant lesions. Adv Anat Pathol 2013;20:227-237.

7 Kusanagi Y, Kojima A, Mikami Y et al. Absence of highrisk human papillomavirus (HPV) detection in endocervical adenocarcinoma with gastric morphology and phenotype. Am J Pathol 2010;177:2169-2175.

8 Park KJ, Kiyokawa T, Soslow RA et al. Unusual endocervical adenocarcinomas: an immunohistochemical analysis with molecular detection of human papillomavirus. Am J Surg Pathol 2011;35:633-646.

9 Ronnett BM, Yemelyanova AV, Vang R et al. Endocervical adenocarcinomas with ovarian metastases: analysis of 29 cases, with emphasis on minimally invasive cervical tumors and the ability of the metastases to simulate primary ovarian neoplasms. Am J Surg Pathol 2008;32:1835-1853.

10 Darragh TM, Colgan TJ, Cox $\mathrm{T}$ et al. The lower anogenital squamous terminology standardization project for HPV-associated lesions: background and consensus recommendations from the College of American Pathologists and the American Society for Colposcopy and Cervical Pathology. Arch Pathol Lab Med 2012;136:1266-1297.

11 Diaz De Vivar A, Roma AA, Park KJ et al. Invasive endocervical adenocarcinoma: proposal for a new pattern-based classification system with significant clinical implications: a multi-institutional study. J Gynecol Pathol 2013;32:592-601.

12 Yemelyanova AY, Vang R, Seidman JD et al. Endocervical adenocarcinomas with prominent endometrial or endomyometrial involvement simulating primary endometrial carcinomas: utility of HPV DNA detection and immunohistochemical expression of p16 and hormone receptors to confirm the cervical origin of the corpus tumor. Am J Surg Pathol 2009;33:914-924.

13 Ansari-Lari MA, Staebler A, Zaino RJ et al. Distinction of endocervical and endometrial adenocarcinomas: immunohistochemical p16 expression correlated with human papillomavirus (HPV) DNA detection. Am J Surg Pathol 2004;28:160-167.

14 Yemelyanova A, Ji H, Shih IM et al. Utility of p16 expression for distinction of uterine serous carcinomas from endometrial endometrioid and endocervical adenocarcinomas: immunohistochemical analysis of 201 cases. Am J Surg Pathol 2009;33:1504-1514.

15 Staebler A, Sherman ME, Zaino RJ et al. Hormone receptor immunohistochemistry and HPV in situ hybridization are useful for distinguishing endocervical and endometrial adenocarcinomas. Am J Surg Pathol 2002;26:998-1006.

16 Jones MA, Young RH, Scully RE. Diffuse laminar endocervical glandular hyperplasia. A benign lesion often confused with adenoma malignum (minimal deviation adenocarcinoma). Am J Surg Pathol 1991;15: 1123-1129.

17 Nucci MR, Clement PB, Young RH. Lobular endocervical glandular hyperplasia, not otherwise specified: a clinicopathologic analysis of thirteen cases of a distinctive pseudoneoplastic lesion and comparison with fourteen cases of adenoma malignum. Am J Surg Pathol 1999;23:886-891.

18 Mikami Y, Hata S, Melamed J et al. Lobular endocervical glandular hyperplasia is a metaplastic process with a pyloric gland phenotype. Histopathology 2001;39: 364-372.

19 Mikami Y, Kiyokawa T, Hata S et al. Gastrointestinal immunophenotype in adenocarcinomas of the uterine cervix and related glandular lesions: a possible link between lobular endocervical glandular hyperplasia/ pyloric gland metaplasia and 'adenoma malignum'. Mod Pathol 2004;17:962-972.

20 Gilks CB, Young RH, Aguirre $\mathrm{P}$ et al. Adenoma malignum (minimal deviation adenocarcinoma) of the uterine cervix. A clinicopathological and immunohistochemical analysis of 26 cases. Am J Surg Pathol. 1989;13:717-729. 